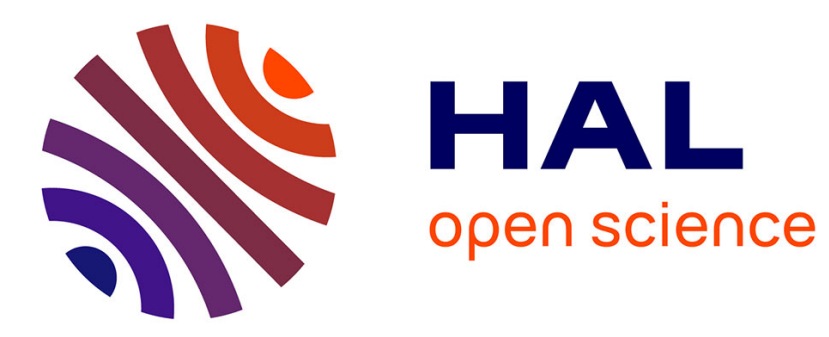

\title{
Structure and magnetic properties of nanocrystalline $\mathrm{PrCo} 3$
}

\author{
Khedidja Younsi, Vincent Russier, Lotfi Bessais
}

\section{To cite this version:}

Khedidja Younsi, Vincent Russier, Lotfi Bessais. Structure and magnetic properties of nanocrystalline PrCo3. Journal of Applied Physics, 2010, 107 (8), pp.083916. 10.1063/1.3388364 . hal-00469737v2

\section{HAL Id: hal-00469737 \\ https://hal.science/hal-00469737v2}

Submitted on 7 Oct 2010

HAL is a multi-disciplinary open access archive for the deposit and dissemination of scientific research documents, whether they are published or not. The documents may come from teaching and research institutions in France or abroad, or from public or private research centers.
L'archive ouverte pluridisciplinaire HAL, est destinée au dépôt et à la diffusion de documents scientifiques de niveau recherche, publiés ou non, émanant des établissements d'enseignement et de recherche français ou étrangers, des laboratoires publics ou privés. 


\title{
Structure and magnetic properties of nanocrystalline $\mathrm{PrCo}_{3}$
}

\author{
K. Younsi, V. Russier, and L. Bessais* \\ CMTR, ICMPE, UMR7182, CNRS - Université Paris 12, \\ 2-8 rue Henri Dunant F-94320 Thiais, France
}

(Dated: October 7, 2010)

\begin{abstract}
The structure and magnetic properties of nanocrystalline $\mathrm{PrCo}_{3}$ prepared by high-energy milling technique have been investigated by means of X-ray diffraction using the Rietveld method coupled to Curie temperature and magnetic measurements. The as-milled samples were subsequently annealed in temperature range from 750 to $1050^{\circ} \mathrm{C}$ for $30 \mathrm{~min}$ to optimize the extrinsic properties. From x-ray studies of magnetic aligned samples, the magnetic anisotropy of this compounds is found uniaxial. The Curie temperature is $349 \mathrm{~K}$ and no saturation reached at room temperature for applied field of $90 \mathrm{kOe}$. The coercive field of $55 \mathrm{kOe}$ and $12 \mathrm{kOe}$ measured at 10 and $293 \mathrm{~K}$ respectively is obtained after annealing at $750^{\circ} \mathrm{C}$ for 30 min suggests that nanocrystalline $\mathrm{PrCo}_{3}$ are interesting candidates in the field of permanent magnets. We have completed this experimental study by simulations in the micromagnetic framework in order to get a qualitative picture of the microstructure effect on the macroscopic magnetization curve. From this simple model calculation, we can suggest that the after annealing the system behaves as magnetically hard crystallites embedded in a weakly magnetized amorphous matrix.
\end{abstract}

PACS numbers: $75.50 . \mathrm{Bb}, 75.50 . \mathrm{Tt}, 76.80 .+\mathrm{y}$

\section{INTRODUCTION}

The intermetallic compounds, $\mathrm{RCO}_{3}(\mathrm{R}=$ rare earth element) have attracted great interest caused mainly by their wide applications as permanent magnets. These compounds present excellent magnetic properties such as large magnetocrystalline anisotropy and important saturation magnetization at room temperature. This interesting magnetic performances of the intermetallic compounds formed by alloying rare earth and $3 d$ transition metals are due to the combination of the complementary characteristics of $3 d$-itinerant and $4 f$-localized magnetism. The coercivity is one of the most important extrinsic properties of a permanent magnet. This properties +depends on the microstructure of the material. Recently nanocrystalline compounds have been extensively investigated, their aim is to find the appropriate nanocrystalline state for a highest coercive field.

The most employed process to prepare these materials are rapid quenching by melt spinning [1] and mechanical alloying [2]. More recently high-energy milling technique, has also been used to produce high coercivity nanocrystalline powders. Rivera-Gómez et al are reported in [3] a high coercivity for $\mathrm{PrCO}_{5}$ compounds milling and annealed at $800^{\circ} \mathrm{C}$ for $1 \mathrm{~min}$ with $H_{\mathrm{c}}=17.3 \mathrm{kOe}$. A high coercivity of $\mathrm{Sm}_{2} \mathrm{Co}_{17}$ nanocrystalline compounds has been obtained by using this method [4] where $H_{\mathrm{c}}=9.6 \mathrm{kOe}$ after milling and annealed at $800^{\circ} \mathrm{C}$ for $30 \mathrm{~min}$.

In this paper, we report the elaboration of nanocrystalline $\mathrm{PrCo}_{3}$ by high-energy milling, and their structural and magnetic properties in amorphous state and after re-

*Author to whom correspondence should be addressed; electronic mail: bessais@glvt-cnrs.fr crystallization. Hard magnetic properties of the as milled $\mathrm{PrCo}_{3}$ have been improved by a controlled nanocrystallization, which optimize the size of grains in order to obtain a high coercivity.

We also propose a simple model for the system after annealing based on a simple lattice of crystallites embedded in an amorphous matrix. The simplest realization of such a model is considered here, in order to bring out at the qualitative level the microstructure effect on the magnetization curve. This model is treated in the framework of micromagnetism, i.e. through a continuous medium type of approach, and the calculations are performed by using the MAGPAR [5] code based on the finite elements method.

\section{EXPERIMENT}

A polycrystalline $\mathrm{PrCo}_{3}$ was prepared by melting highpurity starting elements $(>99.9 \%)$ in an induction furnace under a protective environment( $\mathrm{Ar}$ atmosphere). Their homogeneity was insured with five times consecutive melting. These ingots were used as pre-alloys to manufacture samples by high-energy milling. All powder handling was performed in a glove box under a highpurity $\mathrm{Ar}$ atmosphere with an $\mathrm{O}_{2}$ and $\mathrm{H}_{2} \mathrm{O}$ rate around $1 \mathrm{ppm}$ with vial sealed glove box.

The mixtures were sealed in hardened steel vial together with $15 \mathrm{~mm}$ diameter steel balls. Milling was performed for $5 \mathrm{~h}$ under a high purity Ar atmosphere with a ball to powder ratio of $15: 1$, in a high-energy planetary ball mill Fritsch P7. These milling conditions correspond to kinetic shock energy, shock frequency and injected shock power values respectively equal to $0.81 \mathrm{~J} / \mathrm{hit}, 62 \mathrm{~Hz}$ and $19.5 \mathrm{watt} / \mathrm{g}$. After milling, the powder mixtures was wrapped in tantalum foil and sealed in silica tubes under 
a vacuum of $10^{-6}$ Torr, then annealed for $30 \mathrm{~min}$ at temperature between 750 and $1050^{\circ} \mathrm{C}$ followed by quenching in water.

The purity of the final phase was checked by X-ray diffraction (XRD) using Brucker diffractometer mounted with the Bragg-Brentano geometry and automatic divergence slit, using the $\mathrm{CuK} \alpha$ radiation. The unit parameters were measured with $\mathrm{Si}$ as standard $(a=5.4308 \AA)$ to insure a unit-cell parameter within the accuracy of $\pm 1 \times 10^{-3} \AA$. The counting rate was 22 s per scanning step and step size of $0.04^{\circ}$. To determine the microstructural alloy properties (crystallite size) and crystallographic properties (phase identification and lattice parameters), XRD patterns have been analyzed by Rietveld method.

The data treatment was carried out by a Rietveld refinement $[6,7]$ as implemented in the FULLPROF computer code $[8,9]$ with the assumption of a peak line profile of Thompson-Cox-Hastings allowing multiple phase refinement of each of the coexisting phases [10]. It takes into account the broadening of the diffraction lines and gives, via the Scherrer formula, the autocoherent diffraction domain size. The weight percentage of each of the coexisting phases is directly obtained with the approximation of a Brindley absorption factor equal to 1 . The goodness-of-fit indicators $\mathrm{R}_{B}$ and $\chi^{2}$ are calculated as usual and described earlier [11, 12].

The magnetic anisotropy of $\mathrm{PrCo}_{3}$ was studied using X-ray diffraction. The X-ray diffraction pattern was recorded on powder sample (grain size $<50 \mu \mathrm{m}$ ) oriented under an external magnetic field. The magnetic field was oriented perpendicular to the surface of the sample and the grains are oriented and shape the needles parallel to the field applied, the powder so directed is analyzed by $\mathrm{XRD}$ at room temperature, thus the $\mathrm{x}$-ray pattern gave information about the easy magnetization direction.

Curie temperature $\mathrm{T}_{c}$ was measured on a differential sample magnetometer MANICS in field of $0.1 \mathrm{kOe}$ and heating rate of $10 \mathrm{~K} / \mathrm{min}$ with around $10 \mathrm{mg}$ sample sealed under vacuum in silica tube in order to prevent oxidation under heating. The Curie temperature was determined from the $M-T$ curves by extrapolating linear part of the $M-T$ curves of the intersection with the extended base line. The $M-H$ curves (Magnetic hysteresis measurement) were plotted at $\mathrm{T}=10$ and $293 \mathrm{~K}$ with a Physical Properties Measurement System (PPMS) Quantum Design equipment and a maximum applied field of $90 \mathrm{kOe}$ on sample in epoxy resin.

\section{RESULTS AND DISCUSSION}

\section{A. Structure analysis}

The $\mathrm{PrCo}_{3}$ compounds crystallize in rhombohedral $\mathrm{PuNi}_{3}$-type structure (space group: $R \overline{3} m$ ). The crystal lattice of $\mathrm{PrCo}_{3}$, can be regarded, approximately, as consisting of the consecutive arrangement of alternating blocks of $\mathrm{PrCo}_{5}$ and $\mathrm{PrCo}_{2}$ along the $c$ axis with the ra- tion $1: 2$ (Fig. 1), having almost the same structure as in the corresponding compounds $\mathrm{PrCo}_{5}$ and $\mathrm{PrCo}_{2}$. The unit cell contains two non-equivalent crystallographic site for Pr ions, $3 a$ and $6 c$, and three sites for Co: $3 b, 6 c$ and $18 h$.

The X-ray diffraction (XRD) of the $\mathrm{PrCo}_{3}$ shows that the compound is single phase with the rhombohedral $\mathrm{PuNi}_{3}$-type structure (space group: $R \overline{3} m$ ). Fig. 2 presents the analysis result of XRD pattern of $\mathrm{PrCo}_{3}$ ingot using the Rietveld analysis. The lattice parameters of $\mathrm{PrCo}_{3}$ compound are $a=5.069 \AA$ and $c=24.795 \AA$. The atomic positions, $\mathrm{R}_{B}, \chi^{2}$ factors from Rietveld fit are given in Table 1. These values of structural parameters are in agreement with the results obtained previously by [13]. This ingot is an appropriate precursor for using as pre-alloys to manufacture samples by high-energy milling.

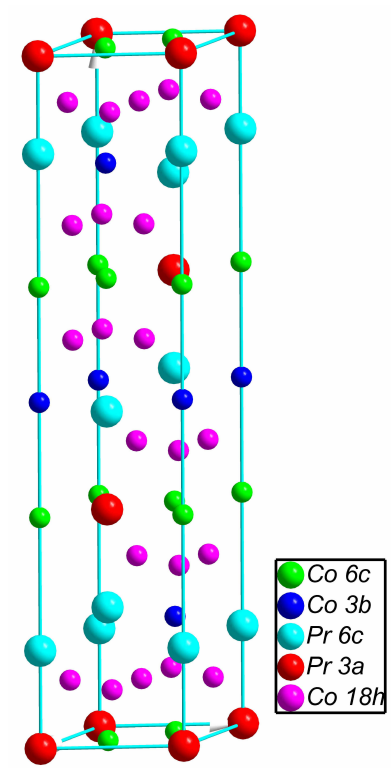

FIG. 1: Crystal structure of $\mathrm{PrCo}_{3}$. Pr atoms occupy the $3 a$ and $6 c$ sites, and Co atoms occupy the $3 b, 6 c$ and $18 h$ sites.

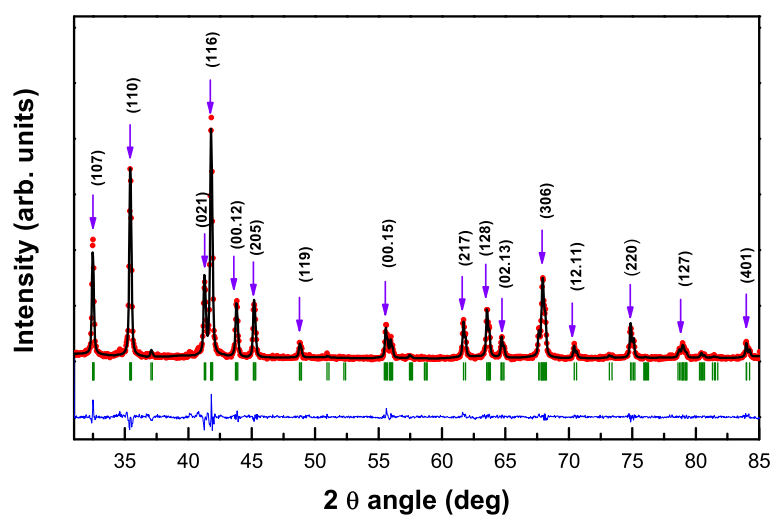

FIG. 2: Rietveld analysis for $\mathrm{PrCo}_{3}$ ingot. 
TABLE I: $a$ and $c$ cell parameters, atomic positions, $\mathrm{R}_{B}$ and $\chi^{2}$ factors from Rietveld fit.

\begin{tabular}{lc}
\hline \hline $\mathrm{a}(\AA)$ & 5.0690 \\
$\mathrm{c}(\AA)$ & 24.7950 \\
$z(6 c) \mathrm{Pr}$ & 0.1414 \\
$z(6 c) \mathrm{Co}$ & 0.3337 \\
$x(18 h) \mathrm{Co}$ & 0.5002 \\
$y(18 h) \mathrm{Co}$ & 0.4998 \\
$z(18 h) \mathrm{Co}$ & 0.0829 \\
$c / a$ & 4.8915 \\
$\mathrm{~V}(\AA)^{3}$ & 551.42 \\
$\mathrm{R}_{B}$ & 5.050 \\
$\chi^{2}$ & 2.300 \\
\hline \hline
\end{tabular}

Figure 3 shows the x-ray diffraction pattern of three samples of $\mathrm{PrCo}_{3}$, the as-milled powder and annealed at 750 and $1000^{\circ} \mathrm{C}$ for $30 \mathrm{~min}$.

The as-milled powder is typical of a quasiamorphous phase and does not show characteristic lines of diffraction of the crystallized state, however, we observe in the low angles a flattened and large line around $42^{\circ}$. Such quasiamorphous matrix suggests the presence of various types of small crystallites. The diffraction peaks become more pronounced with increasing annealing temperature. The annealed samples at 750 and $1000^{\circ} \mathrm{C}$ for $30 \mathrm{~min}$ reveal the presence of $\mathrm{PrCO}_{3}$ phases crystallized. We have observed a small amount (around $0.5 \mathrm{wt} \%$ ) of $\mathrm{Pr}_{2} \mathrm{O}_{3}$ in all samples annealed after milling.

The mean diffraction crystallite size of the powder after annealing at 750 and $1000^{\circ} \mathrm{C}$ for $30 \mathrm{~min}$ obtained by fitting the XRD diagram with Rietveld method, ranges between 31 and $45 \mathrm{~nm}$ respectively, this implies that synthesized materials are noncrystalline.

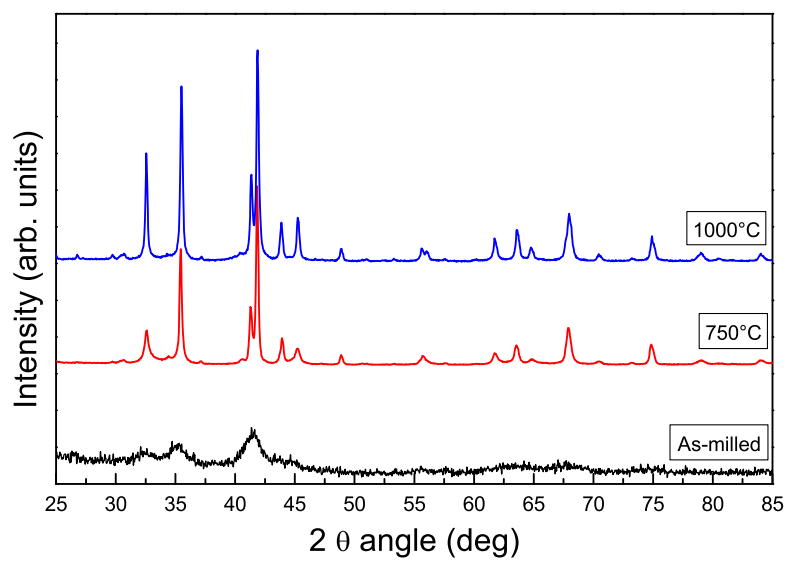

FIG. 3: XRD pattern of the $\mathrm{PrCo}_{3}$ as-milled and annealed samples at 750 and $1000^{\circ} \mathrm{C}$ for $30 \mathrm{~min}$.

\section{B. Intrinsic magnetic properties}

The Curie temperature, also called the magnetic ordering temperature is a direct measure of the exchange interaction, which is the origin of ferromagnetism. This interaction depends markedly on the interatomic distance.

Generally, the Curie temperature in rare-earth transition-metal intermetallic compounds is governed by three kinds of exchange interactions, namely, the $3 d-3 d$ exchange interaction $\left(J_{\mathrm{CoCo}}\right)$ between the magnetic moments of the Co sublattice, $4 f-4 f$ exchange interaction $\left(J_{\mathrm{PrPr}}\right)$ between the magnetic moments within the Pr sublattice, and the intersublattice $3 d-4 f$ exchange interaction $\left(J_{\mathrm{PrCo}}\right)$. The interactions between the rareearth spins $4 f-4 f$ are assumed to be weak and negligible in comparison with the other two types of interactions [14]. There are two exchange interactions in $\mathrm{PrCo}_{3} \mathrm{com}-$ pounds, positive and negative. When the distance of the Co-Co pairs is smaller than $2.45 \AA$, the exchange interactions is negative, whereas at larger Co-Co distances the interaction is positive.

As an example, the thermomagnetic magnetization curve of $\mathrm{PrCo}_{3}$ high-energy milling sample annealed at $750^{\circ} \mathrm{C}$ for $30 \mathrm{~min}$ is presented in Fig. 4. This sample has a unique magnetic phase transition at the Curie temperature of $349 \mathrm{~K}$. This result is in agreement with the data given by Lemaire [15].

The low Curie temperature in $\mathrm{PrCO}_{3}$ compounds is due to the short Co-Co interatomic distances at the sites $6 c-18 h$ and at $18 h-18 h$ (Table 2 ), where the Co atoms couple antiferromagnetically. In this structure, the $6 c-$ $18 h$ and at $18 h-18 h$ interactions are strongly negative.

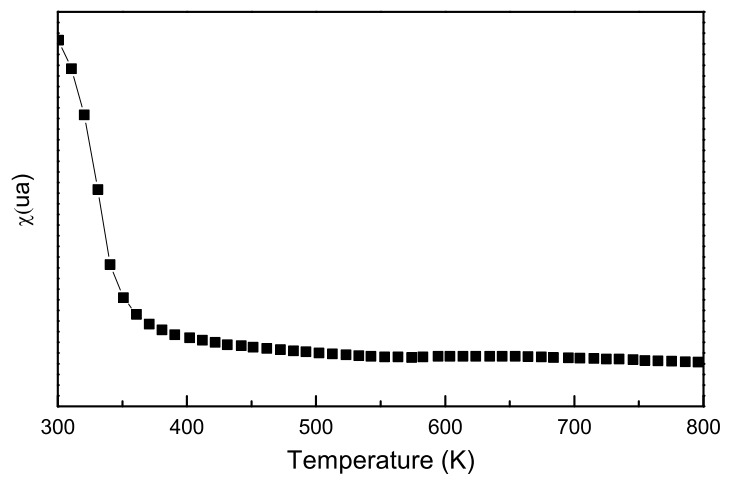

FIG. 4: Thermomagnetization curves of $\mathrm{PrCo}_{3}$ obtained after high-energy milling and annealed at $750^{\circ} \mathrm{C}$ for $30 \mathrm{~min}$.

Isothermal magnetization curves $M-H$ obtained at $293 \mathrm{~K}$ is represented in Fig. 5 for $\mathrm{PrCo}_{3}$. This figure shows that at $293 \mathrm{~K}$ the saturation is not reached, which gives evidence for a magnetocrystalline anisotropy among the highest known in those kinds of systems.

The pattern obtained on sample oriented under an external magnetic field, applied perpendicularly to the plane of the sample, shows only (00l) Bragg peaks 
TABLE II: Interatomic distance $(\AA)$ for $\mathrm{PrCo}_{3}$.

\begin{tabular}{cccccc}
\hline \hline Site & Co $(3 b)$ & Co $(6 c)$ & Co $(18 h)$ & $\operatorname{Pr}(6 a)$ & $\operatorname{Pr}(6 c)$ \\
\hline \hline Co $(3 b)$ & 5.07 & 4.12 & 2.54 & 2.99 & 5.07 \\
Co $(6 c)$ & 4.12 & 2.92 & 2.42 & 4.56 & 2.92 \\
Co $(18 h)$ & 2.53 & 2.42 & 2.41 & 2.92 & 3.50 \\
$\operatorname{Pr}(6 a)$ & 2.99 & 4.56 & 2.99 & 3.18 & 3.50 \\
$\operatorname{Pr}(6 c)$ & 2.99 & 2.92 & 3.26 & 3.50 & 5.07 \\
\hline \hline
\end{tabular}

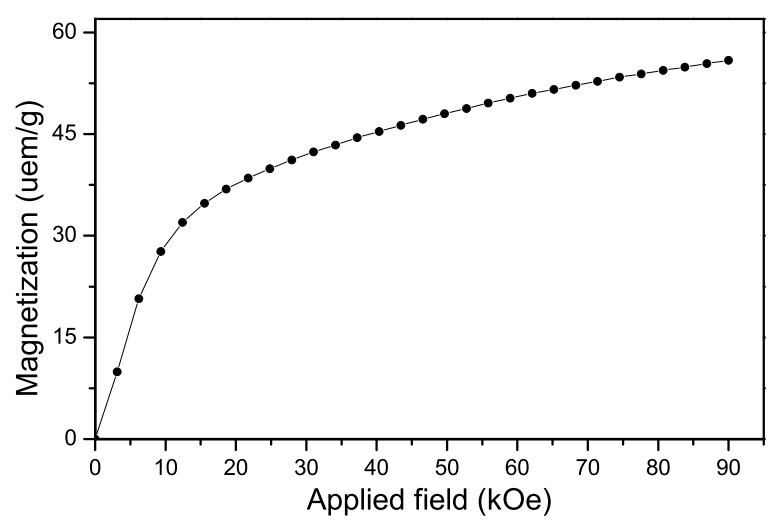

FIG. 5: Magnetization curves of $\mathrm{PrCo}_{3}$ obtained after highenergy milling and annealed at $750^{\circ} \mathrm{C}$ for $30 \mathrm{~min}$, measured at $293 \mathrm{~K}$.

(Fig. 6), which amount to suggest that the anisotropy strengthens the peaks of diffraction of type (00l) and at room temperature the easy magnetization direction is parallel to the c-axis. A similar result is obtained for $\mathrm{YCo}_{3-x} \mathrm{Fe}_{x}$ by [16] obtained by oriented powder under extern field.

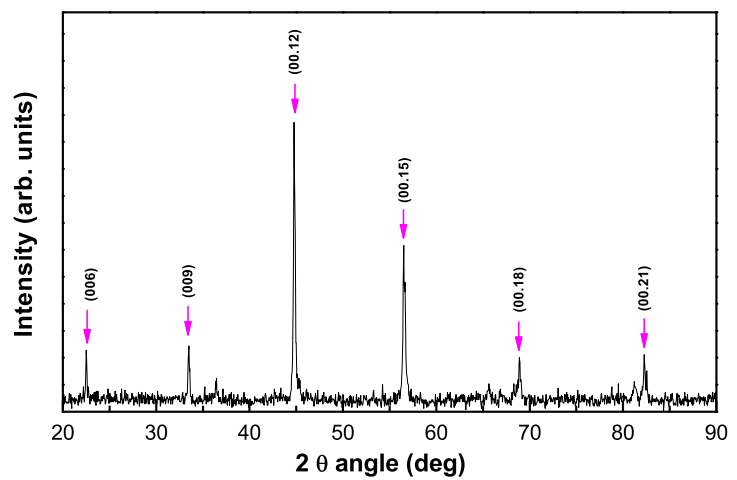

FIG. 6: XRD pattern of $\mathrm{PrCo}_{3}$ oriented.

\section{Extrinsic magnetic properties}

Concerning the extrinsic magnetic properties, we have measured hysteresis loops at 10 and $293 \mathrm{~K}$ for all samples annealed at different temperature $\left(750\right.$ to $1050^{\circ} \mathrm{C}$

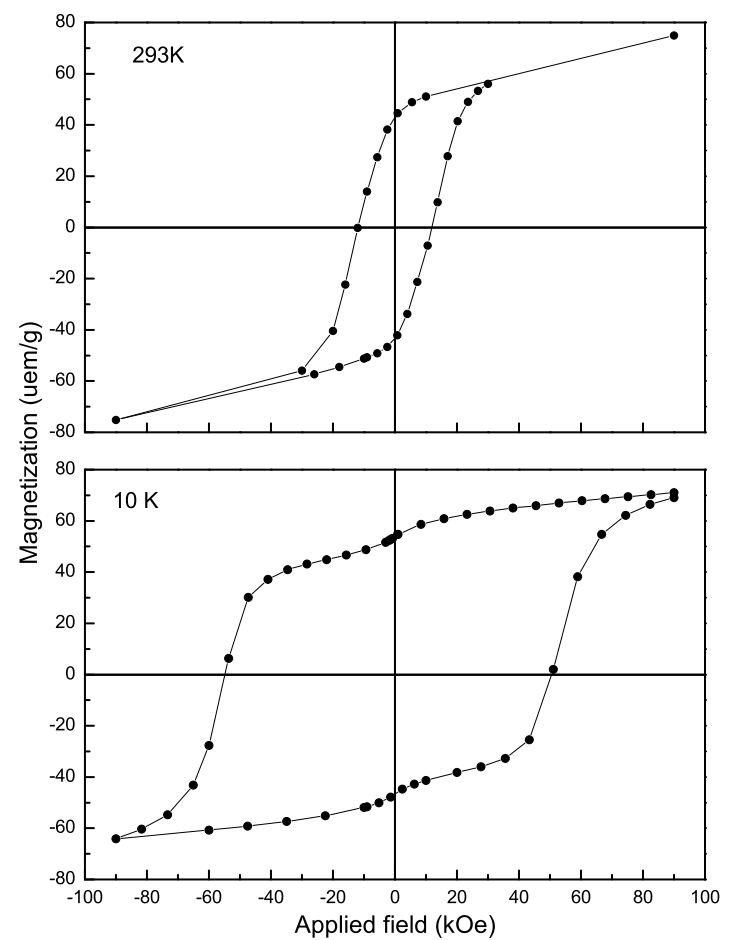

FIG. 7: Hysteresis loop of $\mathrm{PrCo}_{3}$ milling before and after annealing at $750^{\circ} \mathrm{C}$ for $30 \mathrm{~min}$, measured at 293 and $10 \mathrm{~K}$.

for $30 \mathrm{~min}$ ) and plotted $M-H$ curves. Fig. 7 show as an example the hysteresis loops measured at 10 and $293 \mathrm{~K}$ for sample annealed at $750^{\circ} \mathrm{C}$. This sample exhibits high coercivities, we have obtained, for $T=10 \mathrm{~K}$, $H_{\mathrm{c}}=55 \mathrm{kOe}, M_{\mathrm{R}}=56 \mathrm{uem} / \mathrm{g}$ and $M_{\mathrm{R}} / M_{\max }=0.75$. For $\mathrm{T}=293 \mathrm{~K}, H_{\mathrm{c}}=12 \mathrm{kOe}$ and $M_{\mathrm{R}}=44 \mathrm{uem} / \mathrm{g}$ and $M_{\mathrm{R}} / M_{\max }=0.60$ inherent to nanocrystalline state. The coercivity of $55 \mathrm{kOe}$ at $10 \mathrm{~K}$ confirms the large magnetocrystalline anisotropy.

\section{SIMULATION}

In order to get a first picture of the link between the structure at the nanometric scale and the extrinsic magnetic properties at least at the qualitative level, we have performed micromagnetic simulations on a model for our system. Indeed, micromagnetic simulations provide a useful tool for this purpose and have been done for a wide range of nanocomposite magnetic systems [17-22]. It is worth mentioning that quite generally such simulations deal with models for heterogeneous systems including magnetic grains of different materials, typically hard and soft materials, the purpose being to characterize the exchange [17-20] or the magnetostatic coupling between phases [23]. The general finding is that the intergrains exchange coupling leads to a decrease of the coercivity and an increase of remanence when compared to the values of the hard material component. The magne- 
tostatic coupling may be more complex due to the long ranged and the anisotropy nature of dipolar interactions [24]. Here, we model a biphasic system, the phases of which are of the same chemical composition. Indeed, as we have seen in the experimental section, the as milled sample is amorphous, and then after annealing well crystallized grains whose size is controlled by the annealing temperature are formed. Our purpose, rather than to provide a systematic investigation, is to draw out some of the salient features concerning the sample structure which may explain the shape of the magnetization curve. We model the experimental sample as an assembly of well crystallized grains, represented by either spherical or cubic inclusions, embedded in the matrix made of the amorphous phase corresponding to the as milled material. The inclusion shape should be discriminated on physical grounds related to the crystallization growth at the temperature considered. This is beyond the scope of the present work, and we will be lead to discriminate the grain shape from the agreement with experimental results. We emphasize that the main difference between these two cases is the larger relative importance taken by the amorphous matrix, playing the role of grain boundaries, in the model with spherical inclusions.

Since the crystallites are well crystallized, they are characterized by the magnetic constants of the perfect massive crystal. We take the experimental value of the saturation magnetization per unit volume, $J_{s}=0.723 \mathrm{~T}$ as deduced from both the saturation magnetization per unit formula [15], namely $3.8 \mu_{B}$ and the volumic mass as obtained from the measured volume of the unit cell (see table I) which corresponds to $Z=9$. The value of the exchange constant is set equal to that of the archetype of hard material $\left(\mathrm{Nd}_{2} \mathrm{Fe}_{14} \mathrm{~B}\right)$, namely $A_{\text {ex }}=7.7 \times 10^{-12} \mathrm{~J} / \mathrm{m}$ and the magnetocrystalline anisotropy constant is fitted on the experimental value of the coercive field we have got at $\mathrm{T}=10 \mathrm{~K}$, with the result $K_{1}=3.0510^{6} \mathrm{~J} / \mathrm{m}^{3}$. The amorphous matrix is on the one hand non anisotropic $\left(K_{1}=0\right)$, and on the other hand characterized by a very small value of the exchange constant, set somewhat arbitrarily to $A_{e x}=3.8 \times 10^{-13} \mathrm{~J} / \mathrm{m}$. Indeed, since the coercivity remains very high we expect in our two phase model only a negligible exchange coupling between the matrix and the inclusions. Then, concerning the matrix saturation magnetization, we expect a much smaller value than for the crystallized phase of the inclusions [25]; therefore we consider either a non magnetic $\left(J_{s}=0\right)$ or only weakly polarized $\left(0.05 T \leq J_{s} \leq 0.25 T\right)$ matrix. As we shall see these two last points can be justified by the magnetization curve shape. The inclusions have all the same size, while their easy axis, $\hat{n}_{i}$ are distributed either randomly or in a solid angle $\Theta \leq \Theta_{M}$ centered on the direction of the external field, $\hat{h}_{\text {ext }}$ in order to model the effect of the texturation by the field. The influence of the preferential orientation has been investigated only in the case of spherical inclusions. The inclusions are located on the sites of simple cubic lattice. The inclusion packing fraction is therefore limited to values smaller than $\pi / 6$ in the case of spherical inclusions; we set the nearest neighbor distance $d=2.106 R$ close to the minimum value $d=2 R$, which leads to $\varphi=0.445$. We have not such limitation when dealing with cubic inclusions. In this later case the inclusions are separated by a grain boundary of thickness, say $\delta$, and the volume fraction is then $\varphi=(1+\delta / 2 R)^{-3}$ where the inclusion hedge length is set to $2 R$. In the framework of the micromagnetism, the value of $\delta$ cannot be reduced at will since $\delta$ must not reach the microscopisc scale; here we consider either $\delta / d=0.10$ or $\delta / d=0.237$ where $d$ is the minimum intergrain distance which leads to $\varphi=0.73$ or 0.445 respectively. The embedding matrix is a parallepipedic box including 4 layers of 64 lattice sites and the total number of particles taken into account is thus 256. The applied field is set normal to the layers. In order to simulate as far as possible the experimental case, we have chosen $R=17 \mathrm{~nm}$ for the spherical inclusions and $2 R=32.5 \mathrm{~nm}$ for the cubic inclusions. Accordingly the size of the matrix is $288 \mathrm{~nm} \times 288 \mathrm{~nm} \times 144 \mathrm{~nm}$ and in between $297 \mathrm{~nm} \times 297 \mathrm{~nm} \times 158 \mathrm{~nm}$ and $340 \mathrm{~nm} \times 340 \mathrm{~nm} \times 170 \mathrm{~nm}$ in the case of spherical and cubic inclusions respectively. The micromagnetic calculations are performed by using the MAGPAR code [5], based on a finite elements scheme, from either the LLG equation or the total energy minimization. Most of the calculations have been performed with a mesh including 1632000 tetrahedra, and we have checked on a refined mesh of 3159000 tetrahedra the convergence versus the element size of the results in the case of spherical inclusions.

The results for the model with spherical inclusions are displayed in figure (8). First of all, we consider the case of the non magnetic matrix $\left(J_{s}=0\right)$ with randomly oriented particles. As expected, the coercivity takes a large value, namely totally governed by that of the particles, since the exchange constant of the matrix is too low for the particles to be exchanged coupled to and via the matrix. We have checked that even with $J_{s}=0$ a significant value of the exchange constant for the amorphous matrix leads to a decrease of the coercivity, and this as already mentioned justifies our choice of a very small value of $A_{e x}$ for the matrix. However, the main qualitative characteristic which is missing in this first calculated magnetization curve when compared to the experimental one is the small kink in the vicinity of zero external field. Such a kink may be the signature of an uncoupled two phase magnetic behavior. Hence, we have increased the value of the matrix saturation magnetization in order to get such a behavior and we find that $J_{s}=0.05 \mathrm{~T}$ leads to a qualitative agreement with the experimental curve. When the matrix $J_{s}$ value increases the kink amplitude becomes much larger than the experimental one. As a matter of fact, the kink relative amplitude behaves roughly as

$$
\frac{(1-\varphi) J_{s}^{(m a t)}}{(1-\varphi) J_{s}^{(m a t)}+\varphi J_{s}^{(i n c l)}}
$$




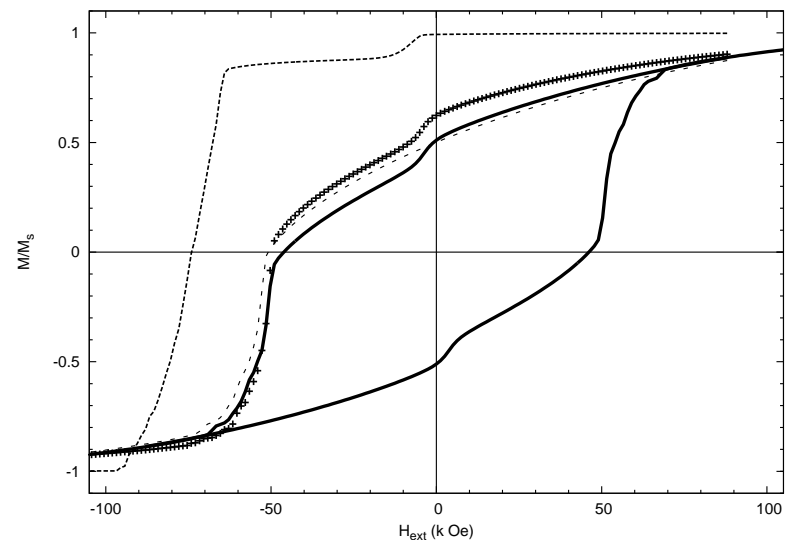

FIG. 8: Calculated de-magnetization curve in terms of the external field for the systems of spherical inclusions with $\varphi=0.45$. Randomly distributed axes and matrix saturation magnetization $J_{s}=0$, dashed line; $J_{s}=0.05 \mathrm{~T}$, solid line. The whole hysteresis curve in this later case is obtained by using the symmetric of the de-magnetization curve. $J_{s}=0.05 \mathrm{~T}$ and $\Theta_{M}=\pi / 2.3$, crosses; $\Theta_{M}=\pi / 15$, dotted line.

The agreement between the calculated hysteresis curve and the experimental one, for randomly distributed axes and $J_{s}^{(m a t)}=0.05 \mathrm{~T}$ is qualitatively satisfying. We have to mention however, that we display in figure (8) the magnetization curve reduced by the total saturation magnetization, $M_{s}$, of the system the value of which, $M_{s}=\varphi J_{s}^{(i n c l)}+(1-\varphi) J_{s}^{(m a t)}$ is significantly smaller than the experimental one. This is due both to the small value of the saturation magnetization of the amorphous matrix obtained to reproduce the amplitude of the kink at $H_{e x}=0$, and to the large fraction of the total volume occupied by the matrix. The model with cubic inclusions can remedy to this drawback since then the inclusion packing fraction is not limited as already mentioned. The results obtained with $\varphi=0.73$ are displayed on figure (9) for the matrix saturation magnetization $J_{s}^{m a t}=0.05 \mathrm{~T}, 0.125 \mathrm{~T}$ and $0.250 \mathrm{~T}$. The first conclusion is that at the qualitative level, the hysteresis curve looks like that of the system with spherical inclusions which may be the consequence of the easy axis random orientation. Now, since the matrix volume fraction is strongly reduced, the amplitude of the magnetisation kink due to the matrix magnetization reversal compares to the experimental one with a larger value for $J_{s}^{m a t}$ than in the model with spherical inclusions, namely $J_{s}^{\text {mat }} \sim 0.125 \mathrm{~T}$. However, increasing the matrix saturation magnetization leads to an important matrix/inclusion magnetostatic coupling resulting in a non negligible reduction of the coercivity (see figure 9). Thus the best agreement with the experimental results is obtained with the model with cubic inclusions, $\varphi=0.73$ and $J_{s}^{(m a t)}=0.125 \mathrm{~T}$ (i.e. $\left.J_{s}^{(m a t)} / J_{s}^{(i n c l)}=0.172\right)$. With this set of parameters, we get for the saturation magnetization of the composite system $J_{s}^{(t o t)}=0.777 J_{s}^{(i n c l)}$. The simulated magnetization curve of the model with cubic inclusions and $\varphi=0.445$ is quite close to the that of the model with spherical inclusions and is thus not shown. We also get an important reduction of the coercivity with the increase of the matrix saturation magnetization.

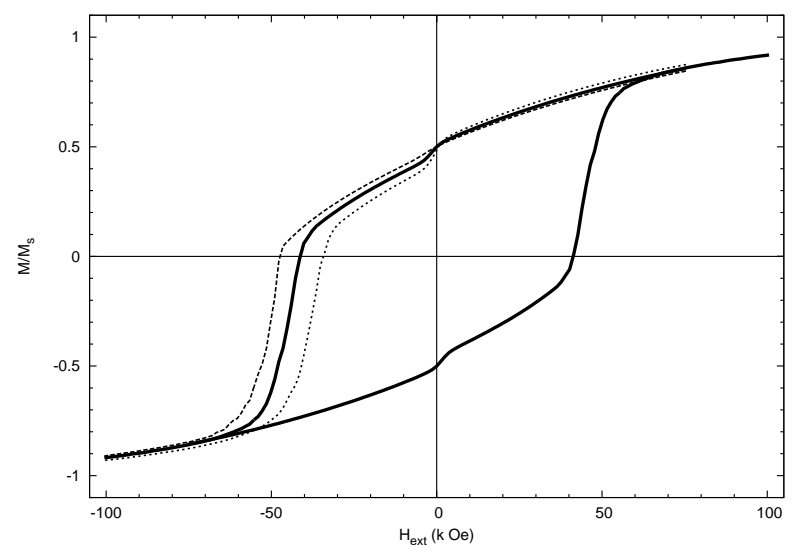

FIG. 9: Calculated de-magnetization curve in terms of the external field for the systems of cubic inclusions with $\varphi=0.73$. Randomly distributed axes and matrix saturation magnetization $J_{s}=0.05 \mathrm{~T}$, dashed line; $J_{s}=0.125 \mathrm{~T}$, solid line and $J_{s}=0.25 \mathrm{~T}$, dotted line.

Then, and only for the model with spherical inclusions, we have examined the effect of the texturation via the easy axes distribution. In this calculation, the maximum value of the polar angle $\Theta$ of the axes $\hat{n}_{i}$ is either $\pi / 15$ or $\pi / 2.3$ for a strong or a very weak preferential orientation respectively. Notice the strong sensitivity to the variation of $\Theta_{M}$; indeed the random distribution corresponds to $\Theta_{M}=\pi / 2$. We emphasize that this orientation effect may represent a consequence of a non spherical shape for the particles. Finally, we have also calculated the magnetization curve for particles of size $R=35 \mathrm{~nm}$ with nearly the same result at least in the case on a non magnetic matrix. Therefore, as expected, we conclude that the strong size effect observed on the coercivity of nanocomposite materials is mainly due to the dependence of the crystallite magnetic properties on the size.

\section{CONCLUSION}

In summary, the $\mathrm{PrCo}_{3}$ intermetallic compound, crystallize in rhombohedral $\mathrm{PuNi}_{3}$-type structure (space group: $R \overline{3} m$ ). This compound is a highly anisotropic uniaxial ferromagnet with the easy magnetization direction is parallel to the c-axis. No saturation reached at room temperature for applied field of $90 \mathrm{kOe}$. High coercivities of about $12 \mathrm{kOe}$ at $293 \mathrm{~K}$ and $55 \mathrm{kOe}$ at $10 \mathrm{~K}$ can be reached in $\mathrm{PrCo}_{3}$ alloy prepared by high-energy milling method followed by annealing at $750^{\circ} \mathrm{C}$ for $30 \mathrm{~min}$. High energy milled method following the controlled nanocrystallization brings high magnetic properties with smaller grain size more adequate to favor an available microstruc- 
ture to perform this properties, so these results show that $\mathrm{PrCo}_{3}$ compounds are promising materials for permanent magnet applications. From the model calculations of the macroscopic extrinsic properties we can propose a simple picture of the microstructure. Indeed, the magnetization curve presents a signature of a system made of two uncoupled phases: the magnetically hard crystallites and the amorphous matrix. The presence of the small kink in the magnetization curve is analyzed as the reversal of the matrix magnetization; the localization of this kink at nearly zero applied field and the its small amplitude are indications that the matrix is, as expected, magnetically very soft and characterized by a small saturation magnetization. This latter point must be taken with care, since here we propose a simple lattice for the crystallite which may lead to an overestimated fraction of the total volume occupied by the matrix also with the model with cubic inclusions. It is important to notice that when dealing with the reduced magnetization, $M / M_{s}$ we find that the influence of the grain shape is rather weak.

\section{Acknowledgements}

Most of the calculations have been performed at the CINES (Centre Informatique National de l'Enseignement Supérieur).
[1] O. Gutfleisch, N. M. Dempsey, A. Yan, K. H. Müller, and D.Givord, J. Magn. Magn. Mater. 272, 647 (2004).

[2] P. G. McCormick, J. Ding, E. H. Feutrill, and R. Street, J. Magn. Magn. Mater. 157, 7 (1996).

[3] F. J. Rivera-Gómez, J. T. Elizalde-Galindo, and J. A. Matutes-Aquino, J. Alloys Compd. 477, 588 (2009).

[4] Z. Chen, X. Meng-Burany, H. Okumura, and G. C. Hadjipanayis, J. Appl. Phys. 87, 3409 (2000).

[5] W. Scholz, T. Schrefl, D. Suess, R. Dittrich, H. Forster, and V. Tsiantos, Comput. Mater. Sci. 28, 366 (2003).

[6] H. Rietveld, Acta Crystallogr 22, 151 (1967).

[7] H. Rietveld, J. Appl. Crystallogr 2, 65 (1969).

[8] J. Rodriguez-Carvajal, M. T. Fernandez-Diaz, and J. L. Martinez, J. Phys.: Condens. Matter 3, 3215 (1991).

[9] J. Rodriguez-Carvajal, Physica B 192, 55 (1993).

[10] J. Rodriguez-Carvajal, M. T. Fernandez-Diaz, and J. L. Martinez, J. Phys.: Condens. Matter 3, 3215 (1991).

[11] C. Djega-Mariadassou and L. Bessais, J. Magn. Magn. Mater. 210, 81 (2000).

[12] L. Bessais and C. Djega-Mariadassou, Phys. Rev. B 63, 054412 (2001).

[13] V. V. Burnasheva, V. V. Klimeshin, V. A. Yartys', and K. N. Semenenko, Inorganic Materials, translated from
Izvestiya Akademii Nauk SSSR, Neorganicheskie Materialy 15, 627 (1979).

[14] D. Givord and R. Lemaire, IEEE Trans. Magn. MAG10, 109 (1974).

[15] R. Lemaire, Cobalt 33, 201 (1966).

[16] M. I. Bartashevich, T. Goto, and K. Koui, Physica B 292, 9 (2000).

[17] R. Fischer, T. Schrefl, H. Kronmüller, and J. Fidler, J. Magn. Magn. Mater. 150, 329 (1995).

[18] R. Fischer and H. Kronmüller, J. Magn. Magn. Mater. 184, 166 (1998).

[19] B. Zheng and S. Zhao, J. Rare Earths 27, 145 (2009).

[20] M. Griffiths, J. Bichop, J. Tucker, and H. Davies, J. Magn. Magn. Mater. 234, 331 (2001).

[21] R. Skomski, J. Phys.: Condens. Matter 15, R841 (2003).

[22] C.-B. Rong, H.-W. Zhang, R.-J. Chen, B.-G. Shen, and S.-L. He, J. Appl. Phys. 100, 123913 (2006).

[23] C.-B. Rong, H.-W. Zhang, R.-J. Chen, S.-L. He, and B.G. Shen, J. Magn. Magn. Mater. 302, 126 (2006).

[24] V. Russier, J. Appl. Phys. 105, 073915 (2009).

[25] S. Sab, L. Bessais, C. Djéga-Mariadassou, N. Dan, and N. Phuc, J. Phys.: Condens. Matter 15, 5615 (2003). 